LA-UR- 91-3572 (FINAL)

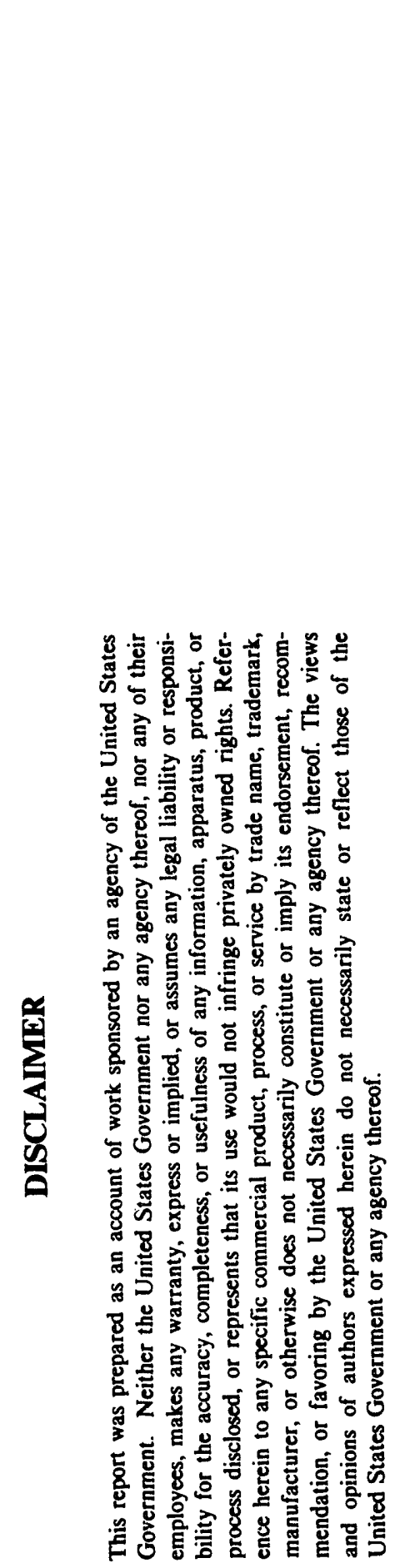

Submitted to:

R. S. Biddle, and P. R. Collinsworth

Author(s):

Title:

A COMPACT NEUTRON DETECTOR FOR A GEOLOGY APPLICATION
IEEE/Nuclear Science Symp, and Medical Imaging Conf. Santa $\mathrm{Fe}, \mathrm{NM}$

November 2-9, 1991

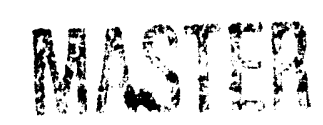

AUG 05 iwis

$09 \mathrm{TI}$

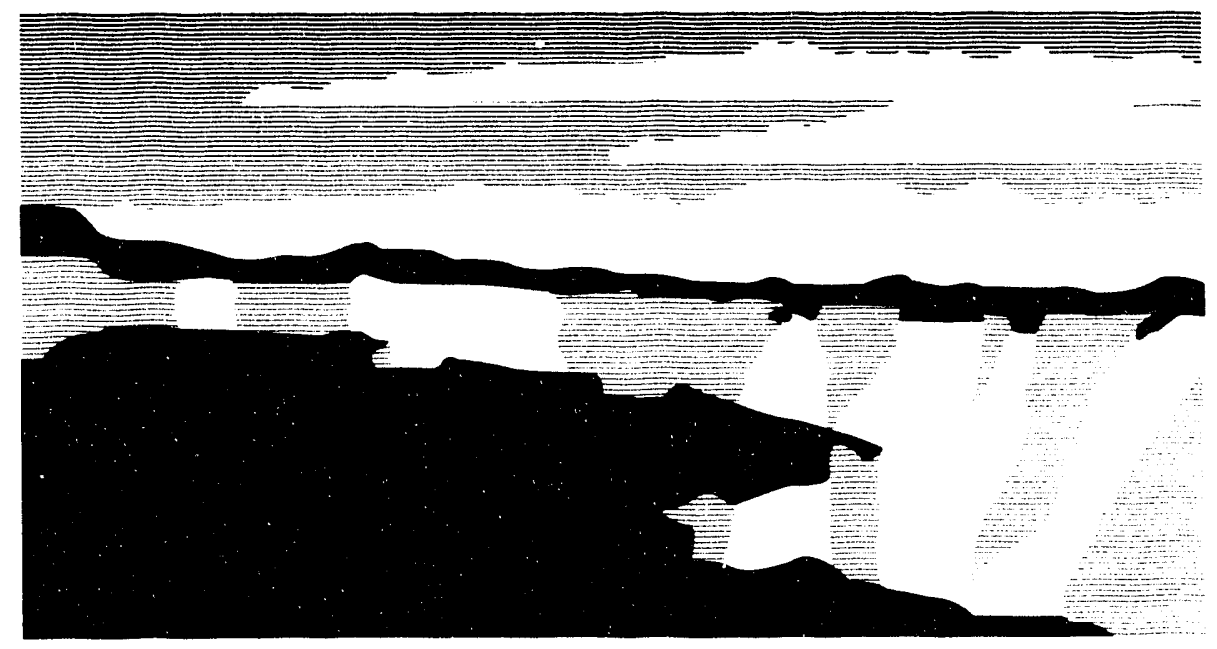

Los Alamos National Laboratory, an affirmative action/equal opportunity employer, is operated by the University of California for the U.S. Department of Energy Linder contract W-7405-ENG-36. By acceptance of this article, the publisher recognizes that the U.S. Government retains a nonexclusive, royalty-free license to publish or reproduce the published form of this contribution, or to allow others to do so, for U.S. Government purposes. The Los Alamos National Laboratory requests that the publisher identify this article as work performed under the auspices of the U.S. Department of Energy. 


\title{
A COMPACT NEUTRON DETECTOR FOR A GEOLOGY APPLICATION
}

\author{
R. S. Biddle and P. R. Collinsworth \\ Safeguards Assay Group, N-1 \\ Los Alamos National Laboratory, \\ Los Alamos, NM 87545 USA
}

Presented at the

1991 IEEE Nuclear Science Sympsoium and Medical Imaging Conference

Santa Fe, New Mexico

November 2-9, 1991 


\title{
A Compact Neutron Detector for a Geology Application
}

\author{
R. S. Biddle and P. R. Collinsworth \\ Safeguards Assay Group, N-1 \\ Los Alamos National Laboratory, \\ Los Alamos, NM 87545 USA
}

\begin{abstract}
We recently designed and built a compact neutron detector for a geology experiment. The detector had to fit inside a 1.5in.-diam borehole in a large block of concrete. We attached a gas-filled, 1-in.-diam ${ }^{3} \mathrm{He}$ tube to a 1 -in.-diam electronics preamplifier package of our design. The electronics package consists of a cylindrically shaped, high-voltage section and a single-channel analyzer with a buffered output. The low-voltage components are mounted on a printed-circuit board. The circuit board and the high-voltage section are attached to a semicylindrical base. The outputs consist of a light-emitting diode for visual observations and a fixed-width, TTL-compatible pulse for a counter. This internal assembly is equipped with coaxial connectors and slips into a thin-walled tube that serves as the preamplifier housing. Power for a detector is supplied by an external, high-voltage supply and a 5-Vdc supply.
\end{abstract}

\section{INTRODUCTION}

We built a compact neutron detector to support a geology experiment. The experiment studied the behavior of cosmic ray nucleons and their secondaries in shallow earth, particularly the effects of air/ground and air/water/ground boundaries. The elemental composition of a concrete block with dimensions $3.7 \mathrm{~m} \times 3.7 \mathrm{~m} \times 1 \mathrm{~m}$ was analyzed and used to simulate the shallow earth. An array of nine horizontal access tubes, each with a $3.8-\mathrm{cm}$ inner diameter, was placed at various depths in the concrete during the construction. A variable, weak, lowenergy neutron flux was to be measured.

The measuring system consisted of 10 compact neutron detectors with a common high-voltage DC power source, up to $2.0 \mathrm{kV}$ for the tube bias and a common 5-V DC power supply for the electronics components. Nine of the detectors were placed in the concrete block; the other was placed in a polyethylene cylinder near the instrumentation rack. This detector was used to normalize the data for shifts in the cosmic-ray flux. The digital outputs were connected to a multiple channel scaler with a common gate and reset commands. A data acquisition computer was used to control and read-out the scaler. The accumulation interval was adjustable, but nominally set for an hour. The detectors were separated from the power supplies and data acquisition components by approximately $50 \mathrm{ft}$ of cable.

A compact neutron detector consists of an electronics module that is directly connected to a gas-filled proportional

This work was partially supported by the US Department of Energy, Office of Safeguards and Security. detector tube. A 2.54-cm-diam tube houses the filter for the externally supplied high voltage and the single channel analyzer (SCA) circuitry. The detector tube interfaces with the electronics module through a male $\mathrm{HN}$-series connector mated with a female HN series end on the detector tube. The maximum diameter dimension for the assembled unit is $2.54 \mathrm{~cm}$. The length of the electronics module is approximately $25 \mathrm{~cm}$. The HN series connector interface allows the detector tube to be easily replaced. Additional moisture barrier protection may be provided in the connector area with tape, shrink tubing, or sealant as necessary because the diameter of the connector is less than the width of the detector tube body. The tubes used for this application were $2.54 \mathrm{~cm}$ in diameter and $30.5 \mathrm{~cm}$ long with 4 atm of ${ }^{3} \mathrm{He}$. They were supplied by the manufacturer as part of a precisely matched set. This design was tested with only this length of detector tube but should be compatible with a broad range of tube lengths.

\section{DESIGN GOALS}

The requirements for the compact detector were as follows: the diameter had to be equal to or less than the detector tube diameter; the detector tubes were to be separate and recoverable from the complete assembly; the high-voltage source could be external; and the design had to be based upon mature, reliable SCA circuitry.

\section{A. Brief Discussion of Design Trade-offs}

1. Compact Detector Diameter. We considered several different support structures for the detector, including a solid outer tube, an inner post, and a split outer shell. A solid outer tube was selected because it offered better grounding, shielding, structural strength, and ease of fabrication. The disadvantages of greater wall thickness, reduced volume, and difficult access to circuitry were countered by using thin-walled tubing with a slip-in cartridge to support the internal components.

2. Recoverable Detector Tubes. We considered several solutions including a male, HN-series bulkhead connector, several different types of between-series adapters and bulkhead connectors, and a specially machined adapter and insulator on the end of the support tube. An HN-male-to-MHV-female adapter was selected to connnect the body of the adapter to the body of the compact detector housing. This solution required the minimum number of components, was readily available, and formed a natural socket to receive the slip-in cartridge. The disadvantage was having to maintain a secure ground and a bond between the adapter, the slip-in cartridge, and the support tube. 
3. External $H V$ Source. Initially, we considered generating the required tube bias internally using a $D C$ to $D C$ converter with a filter network. The advantages were fewer interface cables and a complete detector package. The disadvantages were larger physical size, greater complexity, and increased potential for internally generated noise. Because the objective was to measure a weak flux and therefore a low number of counts, the possibility of introducing internally generated noise made an internal HV source unacceptable.

4. Mature Reliable SCA. The existing SCA circuit design has been used successfully for more than five years with detectors of various lengths and quantities.[1] The standard circuit design allows numerous options and can be configured for many detector-package requirements. The existing circuit is on a rigid printed circuit board that is nominally $3.8 \mathrm{~cm} \mathrm{x} 5.7 \mathrm{~cm}$. This configuration is not compatible with the specification to fit in a $2.54-\mathrm{cm}$ diameter. We decided to miniaturize the existing circuit so that it would fit in the desired diameter and modify the circuit, if necessary, to eliminate options not required in this configuration.

\section{B. Compact Detector Design}

When size is not important, the components and the modules of the neutron detector are housed in rectangular packages with a minimum side dimension of about $7.5 \mathrm{~cm}$. The highvoltage filter section is isolated by a shield or grounded plate, or placed in a separate package. The packages are linked with coaxial cable and connectors.

1. Tubular Housing. The housing provides an electrostatic shield for the electronics module and provides structural strength for the assembly. A male-HN-to-female-MHV adapter provided the interface between the detector tube and the electronics module. The adapter was clamped into the housing using the three set screws on the MHV body of the adapter.

The housing is a $25-\mathrm{cm}$ length of stainless-steel thinwalled tubing with a plug welded into one end (see Fig. 1). An O-ring groove was cut into the face of the plug. The outer shell of the HN portion of the adapter was free to rotate and thus allowed the connector to join with the detector-tube end. We compressed the O-ring mechanically and tightened the clamping screws.

2. HV Filter Capsule. The HV filter consists of three carbon resistors and two capacitors. Two if the resistors and a capacitor form a T-type filter. The other capacitor couples the signal from the detector-tube terminal to the input of the charge amplifier while a parallel resistor connected to ground discharges the capacitor. We selected a high-quality Glassmike capacitor for the signal-coupling capacitor. Although the The physical size was a drawback, the superior low-noise performance was necessary.

The HV capsule was made using a thin-walled, clear acrylic tube with a flat, clear acrylic plug glued in each end. The plugs were pre-drilled to help situate the components in the capsule. After the capsule was assembled and tested, it was filled with a low-conductivity, thermosetting sealant or potting material. This material secured the components in position, provided a seal against moisture, and increased the voltage stand-off until only $0.01 \mathrm{~mm}$ were required to separate the components.

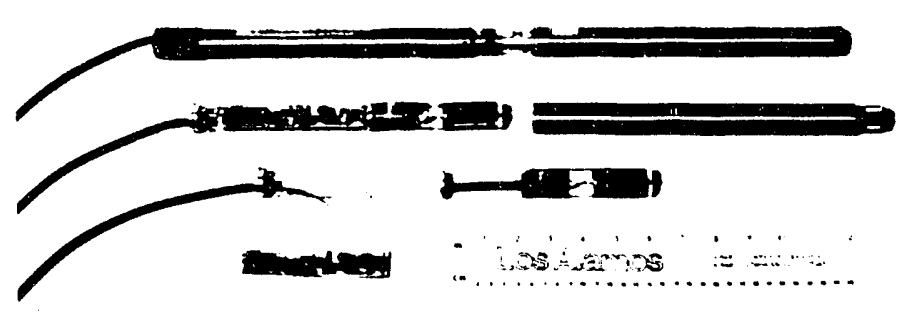

Fig. 1. Disassembled views of compact detector with neuron detector tube.

The external tube bias was delivered to the HV capsule with a coaxial cable. Inside the capsule the cable was terminated in a pigtail configuration. The outer sheath of the cable was epoxied to the acrylic plug to secure the assembly until the potting material could be added and given time to cure.

3. Printed Circuit Board Carrier. The carrier provided a mounting surface and ground plane for the printed circuit board and mounting panels for cable connectors and the HV capsule. The carrier is the primary mechanical component of the insertion capsule. The HV capsule attaches to the front surface with a single screw, and the leads from the capsule are routed through holes in the front face. The coax cable that supplies the high voltage to the capsule is routed through a groove milled along the length of the capsule. This cable exits the carrier through a clearance hole and the cable is terminated with an SHiv connector.

Bulkhead connectors for power and the output signal are mounted in the rear panel along with a light-emitting diode (LED) that indicates a countable event. The rear panel serves as the cover to enclose the tube.

4. Printed Circuit Board. The $2.0 \mathrm{~cm} \times 11.4 \mathrm{~cm}$ circuit board is attached to the carrier. The single tube configuration permitted several functions to be eliminated and reduced the range of opera ing parameters, thus simplifying the task of placing the circ uit in this configuration (see Fig. 2). Some of the circuit modifications are discussed in the the following paragraphs.

Surface Mount Components. All the components were surface mounted except the Amptek charge amplifier, two small-signal diodes and two large inductors used in the power supply filters. These required through-holes.

Single Turn Potentiometer. We retained the threshold adjusting potentiometer, but greatly reduced its range with a series resistor because the tubes were the same length and were well matched for performance. We chose a single-turn, lowprofile open frame potentiometer with about $270^{\circ}$ of rotation for this application. 


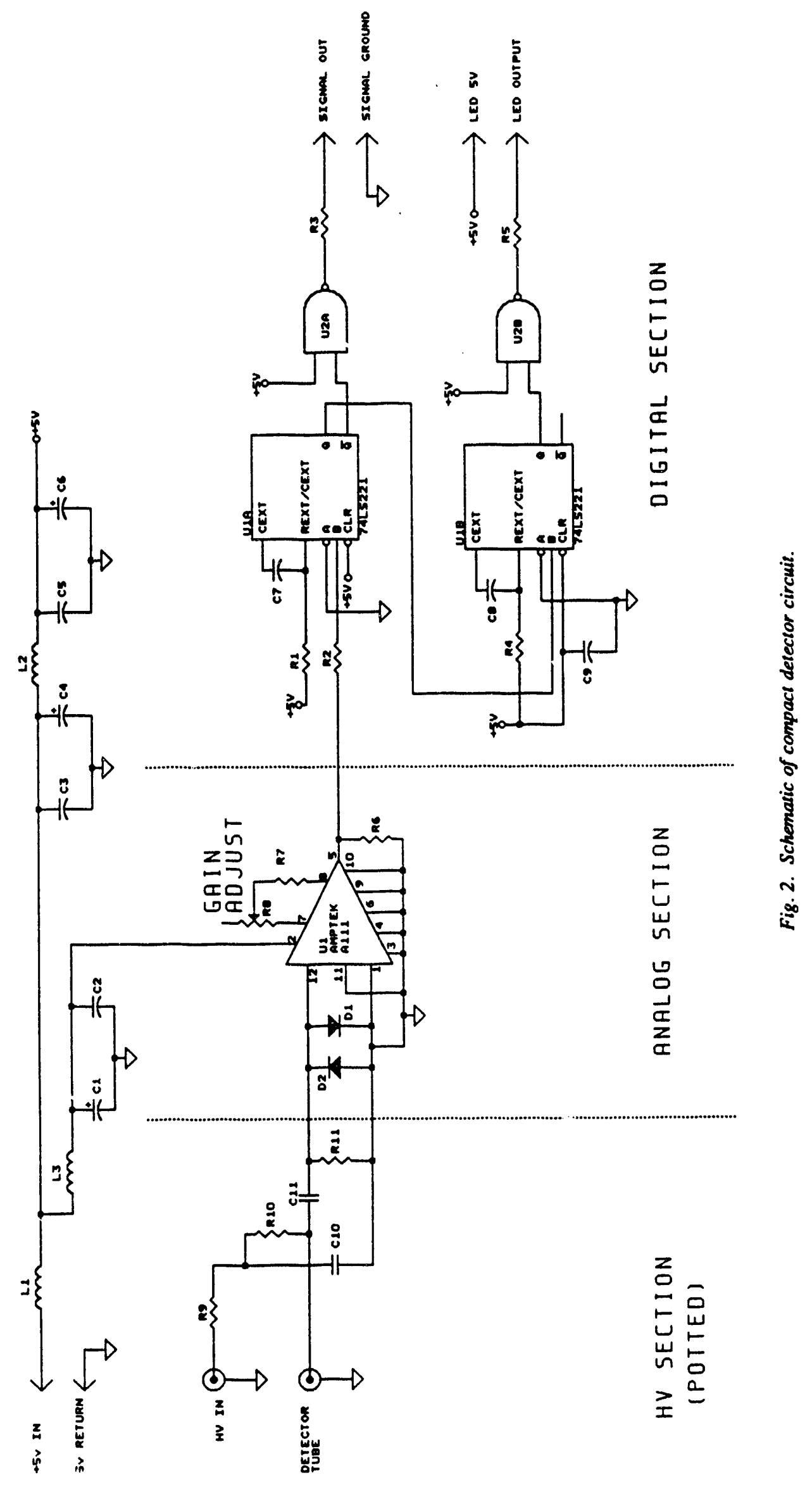


ENABLE/OR Circuit Elimination. The unitary nature of the detector allowed us to simplify the circuitry somewhat. The compact detector would only be operated with a single detector wbe and each unit would be connected to an individual scaler channel. Therefore the OR circuit was not required. Also the ENABLE signal on a dedicated single unit system did not make sense, so we eliminated these two functions. The unit is best disabled by disconnecting the input power.

Fixed Width Output Pulse. Standard circuit designs use a variable-width output pulse to adjust the dead-time parameter for balance when summing multiple detectors. In the geology experiment, each compact detector is on a single channel, so we selected a fixed-width output pulse of $50 \pm 5 \mathrm{~ns}$.

5. Insertion Cartridge. An insertion cartridge was formed by attaching the HV capsule to the carrier for the printed circuit board. The power and output signals were common with the circuit board ground to which the carrier was attached. The internal components in the HV capsule were referenced to the circuit board ground. The detector tube end of the HV capsule was equipped with a gold-plated coaxial connector pin that slipped into the MHV portion of the adapter that mated with the detector tube. The circuit carrier was secured to the outer case with two screws. In this configuration the outer case of the compact detector was a pari of the ground path. This technique provided a ground path that was secured by two screws but did not rely on a friction joint.

\section{OPERATIONS.}

Between late September and early October 1990, the detector system was calibrated in the laboratory and then transferred to the persons performing the measurements. From November 6 until December 19,1990, measurements were performed with nine tubes at various depths in the concrete earth simulator and one tube in the equipment rack. The tubes in the concrete measured the secondary neutrons induced by cosmic-rays nucleon, while the rack mounted tube, which was in a polyethylene cylinder, was used to measure shifts in the cosmic-ray flux. Measurements were performed with and without cadmium "wrappers" on the ${ }^{3} \mathrm{He}$ tubes to establish the equilibrium depth of high-energy (epi-cadmium) and thermal neutrons. Some in-situ measurements using a plutonium-beryllium neutron source were also performed; these measurements assured uniformity of response from the detector system.

The initial measurement series was performed without cadmium. There were a few spurious (noise-related) totalized counts in the reference channel during this series. The second series was done with cadmium and produced spurious readings in the reference channel and concrete channel 7 . The third series duplicated the first (noncadmium) series and produced one suspicious reading in concrete channel 7 . The fourth series was the plutonium-beryllium test series, which we have not analyzed for problems. The fifth series repeated the noncadmium test, and again, channel 7 displayed spurious readings. A sixth series was then done with an additional $15 \mathrm{~cm}$ of concrete above the original surface, with and without cadmium wrappers. The experimenters made 161 hourly data records in this configuration; seven displayed a spurious reading in the reference channel. The experiment was then configured to simulate a boulder on the planar surface of the concrete earth simulator by constructing a pyramid of concrete blocks on the slab. The detectors were moved to the slab surface and into the concrete pyramid, and data were collected with and without cadmium. Concrete channel 2 started to produce error readings during this measurement series and essentially became unreliable for the remainder of the experiment (19 days). The reference channel usually displayed a spurious reading on most of the remaining days as well, but at a lesser rate than channel 2. That is, the reference channel would produce several hours of reasonable data, then an hour or more of error data, and then repeat reasonable data, and so on. The original measurement series was rerun for several days after the boulder simulation, with results similar to the original series. The experimenters then simulated snow cover on the slab with a plastic wading pool filled with 6 to 8 in. of water. Again, the detectors were operated with and without cadmium. The original series was then rerun, followed by a plutonium-beryllium test series and another original series duplicate.

The general result of the experiment was a mixed set of reliable and faulty data. During the experiment, most of the slab mounted detectors recorded rates of about 1000 to 2000 counts per hour with the rate being nonlinear with depth into the slab. The initial rates near the surface were low, then increased, and then decreased with increasing depth into the slab with the maximum rates found at about a $20-\mathrm{cm}$ depth. With the cadmium sleeves in place, the rates dropped by about a factor of 10 . Stability in most of the counting data made it simple to detect spurious or error readings. Although two of the detector packages had been faulty on most days, the other eight units displayed only occasional spurious counts or none at all. These detectors provided the experimenters with sufficient data to analyze and a stable baseline with which to detect erroneous data.

At the end of the measurement period, we were provided with a copy of the collected data and an environmental history (weather data) from a nearby meteorological station. Altogether, the system provided more than 900 hours of data. Because problems had been reported with several of the detector packages, with two packages (reference and concrete channel 2) displaying noise problems in almost half the data collection days, we performed a series of diagnostic tests. We operated the detector set on our laboratory bench for several days, in a "transportainer" for another few days, and then in an environmental chamber (with both temperature and humidity being controllable variables). The original counting and weather data and bench, transportainer, and environmental chamber tests allowed us to determine the primary source of the spurious data. Several days of operation on the bench showed that the detectors did not produce high or spurious data rates in the conditions found in our laboratory, which has a nearly constant environment in comparison to the original measurement environment. (The concrete block was located out of doors, near a waste treatment plant setting pond, and the non-detector portion of the data collection system was in a sealable electronics rack in an unheated equipment storage shed.) The transportainer tests also showed no particular spurious counting problem and demonstrated no sensitivity to the diumal temperature cycle.

Our bench and transportainer tests did show that we had no problems with mechanical, transient-induced noise ("microphonic"), and also showed that the detectur set was sensitive to changes in the local neutron flux. In the laboratory we used ad hoc methods of generating transients and in the transportainer, the detector set was subject to regular daily 
vehicle traffic. In neither case did we see anything extraordinary. By striking a ${ }^{3} \mathrm{He}$ tube sharply enough, one can always induce transient noise, but striking the electronics assembly or the mechanical fixture in which the detector set was mounted produced no noise and traffic "rumble" also produced no noise. In the laboratory, the detector set was able to "see" the movement of a neutron source from a well-shielded to a less wellshielded location at a distance of $25 \mathrm{~m}$ overhead with an intervening $2-\mathrm{m}$ concrete ceiling. In the transportainer, the detector set saw several operations involving transfers of neutron sources in the immediate vicinity (within $5 \mathrm{~m}$ of the detectors) (see Fig. 3).

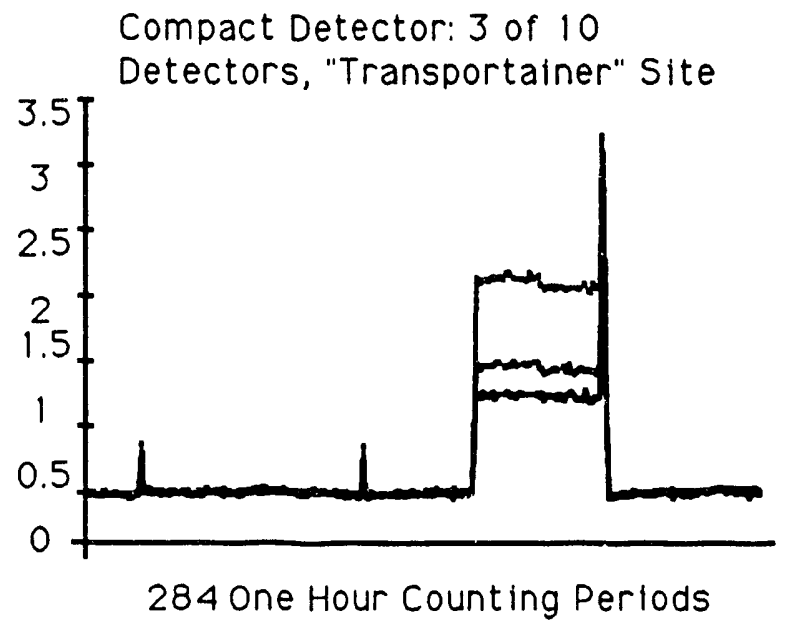

Fig. 3. Detection of source motion.

Environmental chamber testing also showed that temperature sensitivity was not our problem. The detectors do not show a measurable, temperature-related rate of change in the data we have. This is not to claim that there is not a temperature coefficient, but that our data are not sufficient for determining the coefficient. We need more counting data, at a higher counting rate, with a larger deviation in temperature to accurately and precisely determine the temperature coefficient. The two detectors that produced most of our error data did produce noise in conjunction with increasing humidity. As humidity was increased in the test chamber, count rates in the error producing detectors increased in step function fashion (see Fig. 4). An analysis of the spurious data from the measurement operations confirmed the correlation. A correlation matrix was generated using minima and maxima of atmospheric pressure, ambient temperature, relative humidity, and count rates for one of the "bad" detectors. There was a negative or weak (nearly neutral) correlation between temperature (or pressure) and count rates, and a positive correlation between humidity and count rates.

\section{Rate \\ Counting Data vs. Increasing Humidity}

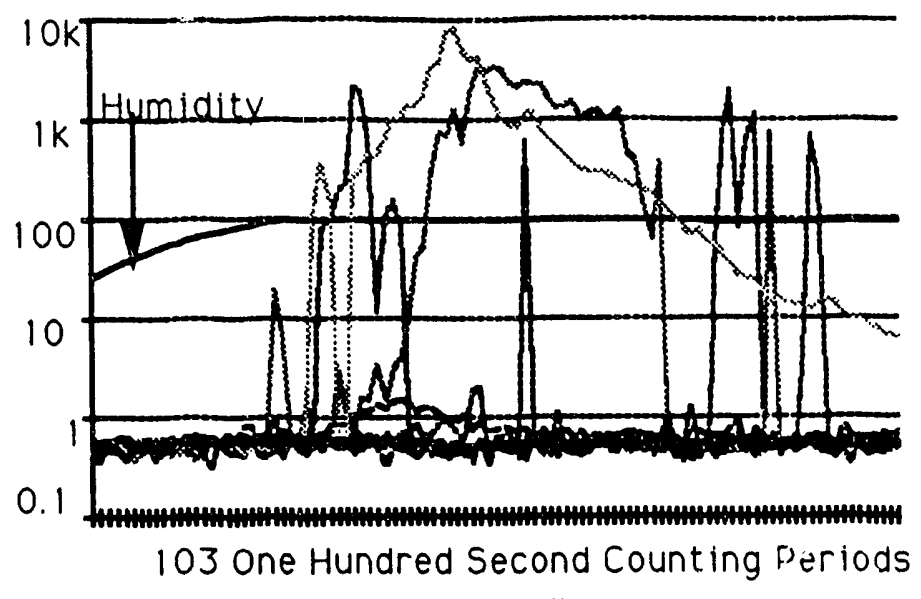

Fig. 4. Effect of increasing humdidity on count rate.

\section{REMEDIES}

A review of the data and the correlations suggested two potential scenarios that could not be repeated in the laboratory. The first scenario was that the local humidity was significantly different from the meteorological humidity because of the large source of moisture in the settling pond. The second scenario was that the set screws in the brass became loose because of the differences in the thermal expansion of the brass compared to the scainless steel. It was speculated that the brass exp2zded, deformed against the set screw, and then loosened upon contraction. Two remedies were applied. A conductive rubberized sealant was applied between the face of the tubing plug and the body of the HN-to-MHV adapter. This look the place of the $O$ ' ring and formed a watertight flexible seal. The second remedy was to place shrinkable tubing on the output end of the housing where the cartridge was inserted and secured. Since these remedies were applied, testing has not been sufficient to demonstrate the spurious count rates.

\section{ACKNOWLEDGMENTS}

We would like to thank the following people for their help: June Fabryka-Martin and Malcom M. Fowler, for proposing and performing the geology experiment; Kenneth E. Kroncke, for the mechanical design of the stainless-steel housing, the printed circuit board carrier, and the high-voltage capsule; Ted Dye and David C. Garcia, for the mechanical fabrication; and Margaret Rivera, for wiring and final assembly of prototype and subsequent units.

\section{REFERENCES}

[1] J. E. Swansen, "Deadtime Reduction in Thermal Neutron Coincidence Counter," Nuclear Instruments and Methods in Physics Research, B(9), 80 (1988). 

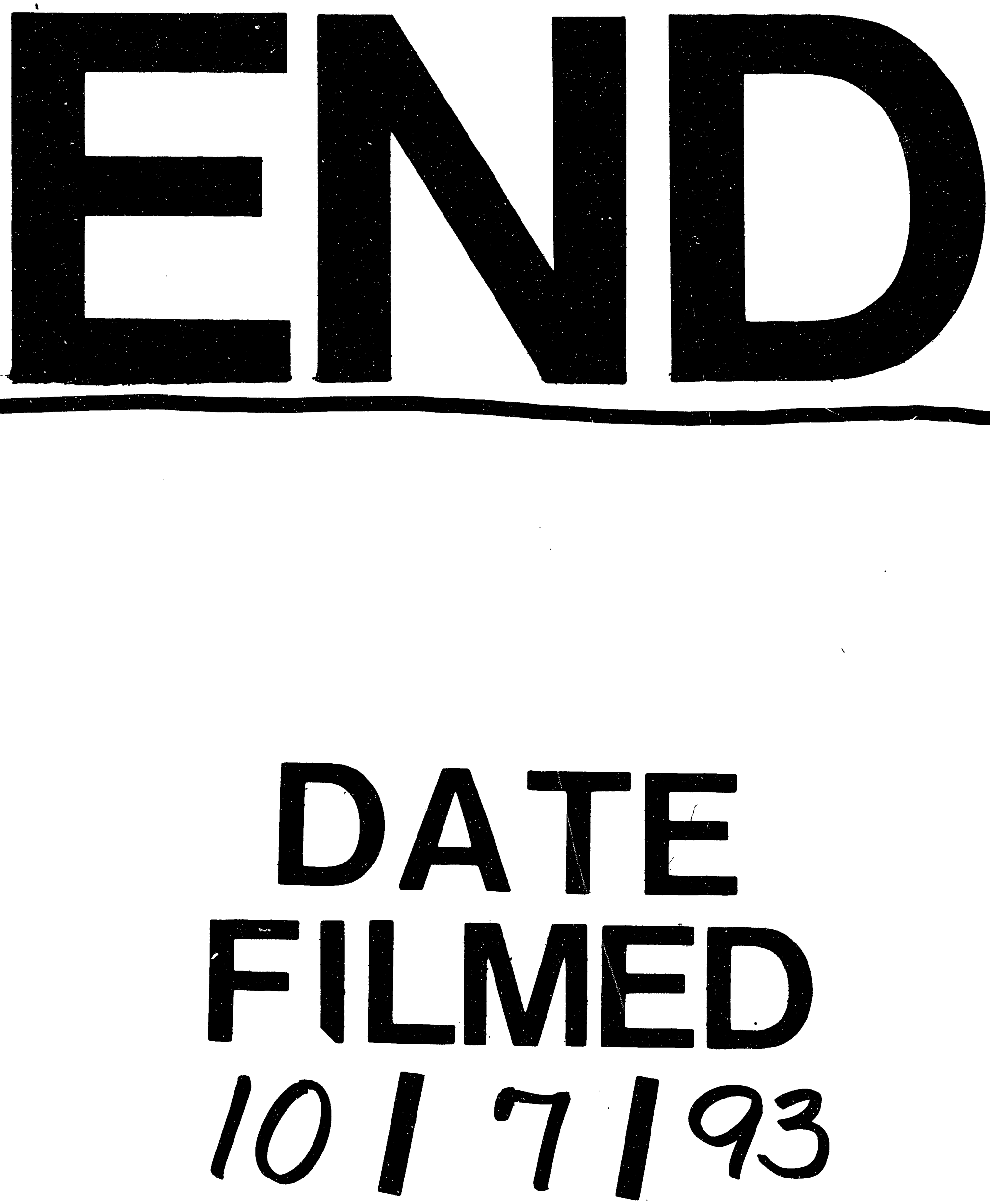


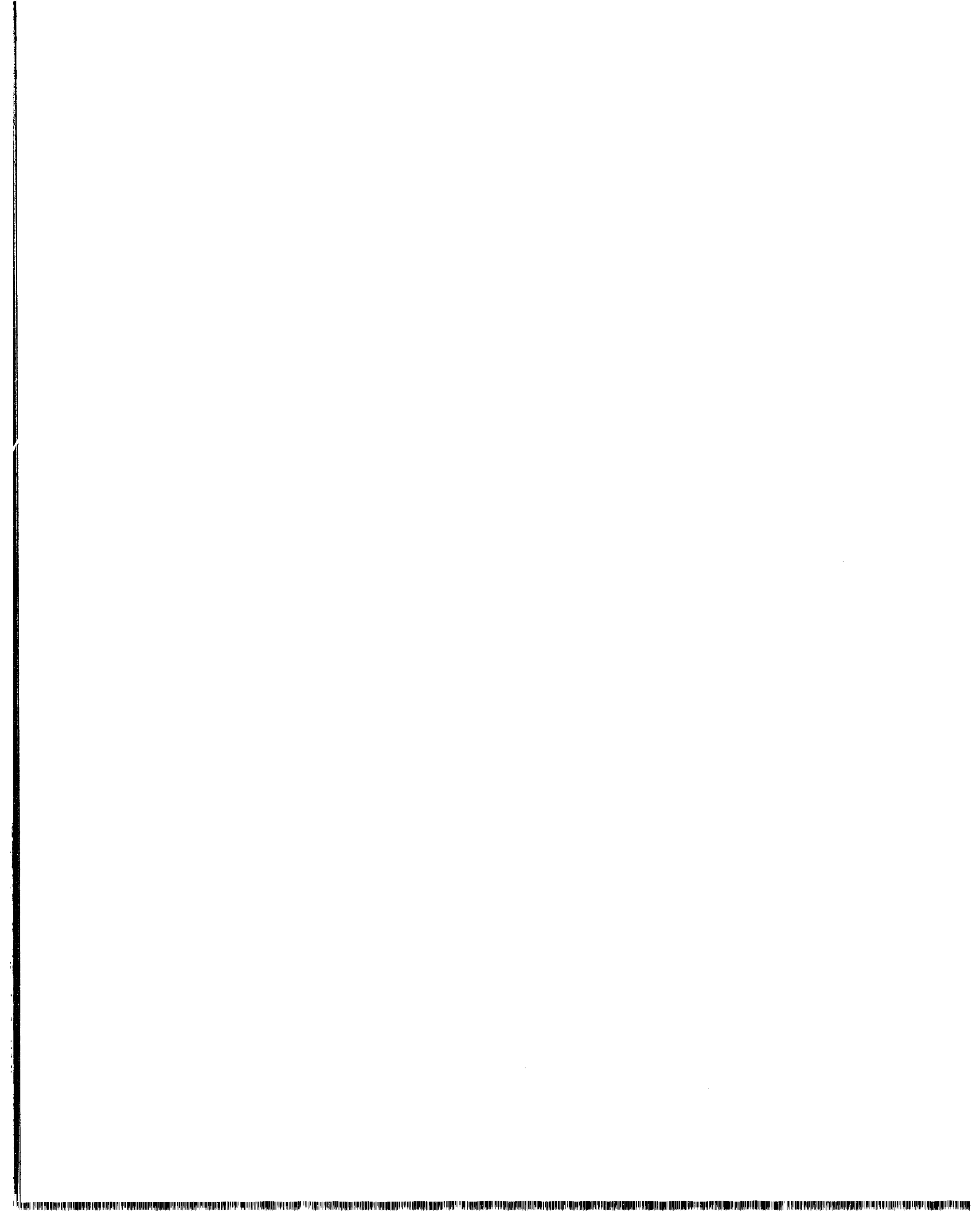

